\title{
Natriuretic Peptides and Assessment of Cardiovascular Disease Risk in Asymptomatic Persons
}

\author{
Lori B. Daniels
}

Published online: 17 February 2010

(C) The Author(s) 2010. This article is published with open access at Springerlink.com

\begin{abstract}
Current tools for cardiovascular disease (CVD) risk assessment in asymptomatic individuals are imperfect. Preventive measures aimed only at individuals deemed high risk by current algorithms neglect large numbers of low-risk and intermediate-risk individuals who are destined to develop CVD and who would benefit from early and aggressive treatment. Natriuretic peptides have the potential both to identify individuals at risk for future cardiovascular events and to help detect subclinical CVD. Choosing the appropriate subpopulation to target for natriuretic peptide testing will help maximize the performance and the cost effectiveness. The combined use of multiple risk markers, including biomarkers, genetic testing, and imaging or other noninvasive measures of risk, offers promise for further refining risk assessment algorithms. Recent studies have highlighted the utility of natriuretic peptides for preoperative risk stratification; however, cost effectiveness and outcomes studies are needed to affirm this and other uses of natriuretic peptides for cardiovascular risk assessment in asymptomatic individuals.
\end{abstract}

Keywords Natriuretic peptides · Risk assessment .

Primary prevention $\cdot$ Screening

\section{Introduction}

Successful prevention of cardiovascular disease (CVD) is predicated on accurate risk assessment to identify individuals most likely to benefit from specific interventions.

\section{B. Daniels $(\triangle)$}

Division of Cardiology, University of California, San Diego,

MC 0986, 9350 Campus Point Drive, Suite 1D,

La Jolla, CA 92037-1300, USA

e-mail: loridaniels@ucsd.edu
Consensus statements regarding prevention of CVD therefore direct significant focus upon their risk assessment recommendations. Whereas older guidelines often focused exclusively on traditional cardiovascular risk factors for assessment of risk [1], recent consensus statements have begun to note the shortcomings of relying solely on this approach while heralding the potential of novel assessment methods, including the use of subclinical measures of disease and cardiovascular biomarkers [2].

Traditional cardiovascular risk factors, although certainly important to incorporate into any risk assessment, are not sufficient for assessing CVD risk. According to the Third National Health and Nutrition Examination Survey (NHANES-3), over 99\% of men and women between the ages of 35 and 74 years have at least one suboptimal risk factor [3]. It is not surprising, then, that major coronary heart disease (CHD) risk factors are highly prevalent even among individuals who never develop heart disease [4]. At the same time, traditional risk factors do not fully explain cardiovascular risk $[5,6]$. The poor specificity and suboptimal predictive value of traditional risk factors, in a setting where early detection of subclinical CVD could lead to prevention of future problems with early treatment, points to the need for better ways to predict cardiovascular risk in asymptomatic individuals.

Cardiovascular biomarkers are emerging as valuable tools in assisting with disease prognosis and risk stratification for a variety of cardiovascular conditions. The natriuretic peptides (NPs), including B-type natriuretic peptide (BNP) and the N-terminal fragment of pro-BNP (NT-proBNP), are released in the setting of myocardial strain and are well established for aiding in the diagnosis, prognosis, and monitoring of heart failure patients [7]. Emerging data suggest that NPs also have predictive value in a variety of other settings, including apparently healthy 
community dwellers, in whom elevated levels are associated with increased risk of cardiovascular morbidity and mortality and may reflect subclinical myocardial or valvular disease $[8,9]$. They are therefore good candidate markers for evaluating asymptomatic individuals who may have subclinical cardiovascular abnormalities or who may be at risk for future cardiovascular events.

\section{Shortcomings of Currently Used Tools for Cardiovascular Disease Risk Assessment}

Tools commonly used for CVD risk assessment are often based on measurement of traditional risk factors, such as blood pressure, cholesterol, and smoking status, and have several limitations. The National Cholesterol Education Program Adult Treatment Panel III risk assessment [10] and the Framingham risk score [11] are two such tools that, although well validated and useful for thinking about risk on a population level, have a number of shortcomings when used in the individual patient. By principally targeting individuals deemed "high risk" by current assessment tools, we are still missing the majority of people who will ultimately develop CVD. To paraphrase a classic paradox, the large number of people at low risk for CVD may give rise to more cases of CVD than the small number who are at a high risk [12].

The paradigm of treating high-risk patients by lowering their blood pressure and cholesterol to a prespecified target level also neglects the fact that regardless of the starting value, for any given absolute change in blood pressure or cholesterol there is a constant relative change in cardiovascular risk (Fig. 1) [13]. Thus, if we could more accurately identify individuals at risk for CVD, aggressive treatment may help prevent CVD even among individuals without marked hypertension or dyslipidemia.

Other shortcomings of global risk assessment tools include an underestimation of life-time risk, poorer estimation of risk in ethnic minority populations, a focus on only CHD events rather than the full spectrum of CVD events worthy of prevention (which includes cerebrovascular disease and peripheral vascular disease in addition to CHD), and the documentation of subclinical disease among many individuals (especially women) whose score classifies them as being at low risk [2,14].

\section{Natriuretic Peptides for Risk Assessment and Cardiovascular Screening}

\section{BNP Versus NT-proBNP}

Although they tend to be well correlated, absolute levels of the two NPs are not interchangeable, and it is helpful for

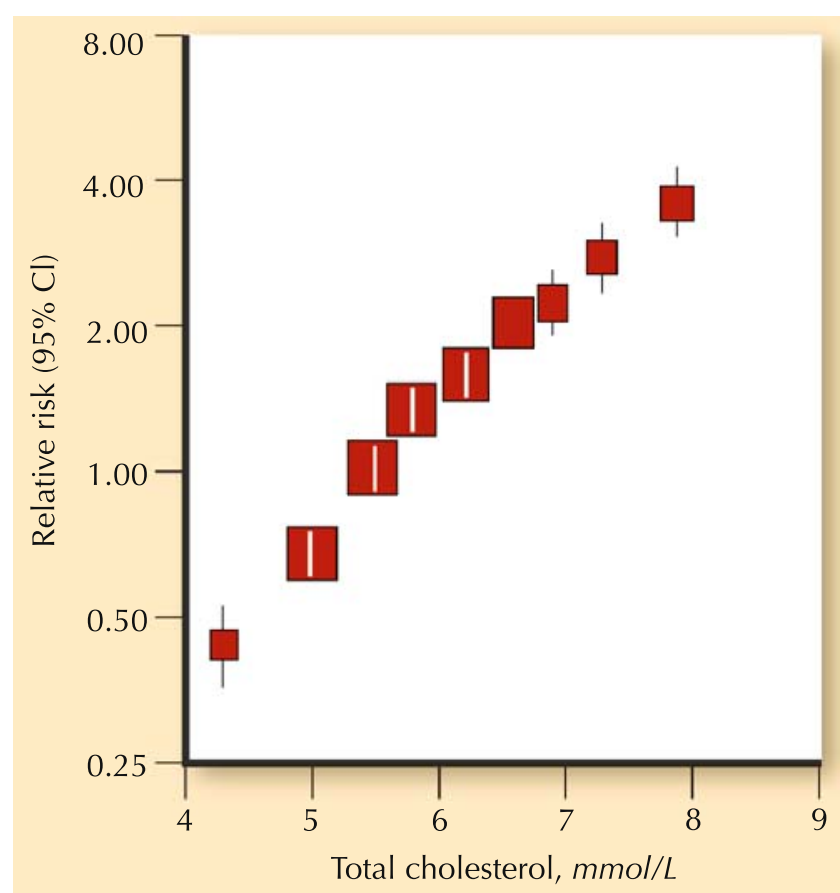

Fig. 1 Relative risk of coronary heart disease (CHD) by cholesterol concentration. (Adapted from Jackson et al. [13]; with permission.)

clinicians to understand the differences and nuances between them. On the occasions where there is a discrepancy in relative levels, personal experience and some data have suggested that it tends to be the NT-proBNP that is the proportionally higher of the two values, which may in part reflect its longer half-life compared with BNP. In this small proportion of cases where the two tests disagree, NTproBNP may perform better [15•].

Performance characteristics for BNP and NT-proBNP are similar in many clinical scenarios, including risk assessment and cardiovascular screening [16]. However, some studies have suggested that NT-proBNP may perform marginally better than BNP in predicting mortality in the community $[17,18]$ and in screening asymptomatic individuals for structural heart disease $[15 \bullet, 19]$.

Natriuretic Peptides and Cardiovascular Disease Risk Assessment

Enthusiasm for using NPs for assessment of CVD risk has grown out of a number of community-based studies showing that levels are predictive of future cardiovascular morbidity and mortality, even among apparently healthy individuals. For example, the Framingham Offspring Study prospectively measured BNP levels in 3,346 asymptomatic middle-aged participants and found them to be independently predictive of death, heart failure, stroke or transient ischemic attack, and atrial fibrillation, even after adjusting for traditional risk factors [8]. Participants with BNP levels 
above the 80 th percentile $(\sim 20 \mathrm{pg} / \mathrm{mL})$ had a $62 \%$ increased risk of death and a $76 \%$ increased risk of a first major cardiovascular event. With each increase in standard deviation in $\log$ BNP levels, there was a $27 \%$ increased risk of death, a $77 \%$ increased risk of heart failure, a $66 \%$ increased risk of atrial fibrillation, and a $53 \%$ increased risk of stroke or transient ischemic attack. NT-proBNP fared similarly in a Danish community-based study where levels were predictive of mortality and first major cardiovascular event (nonfatal myocardial infarction, fatal CHD, unstable angina, heart failure, stroke, and transient ischemic attack) and provided prognostic information beyond traditional risk factors [20]. These findings have since been replicated in numerous studies [9, 21-28]. Thus, NPs may serve as an early warning sign, alerting asymptomatic individuals to their increased risk of CVD.

\section{A Combined Approach: Screening for Subclinical CVD Plus Risk Assessment}

One distinct advantage of using NPs for cardiovascular risk assessment is that in addition to helping predict future cardiovascular events, NPs may also help to screen for prevalent subclinical cardiovascular disease. Important considerations for a successful population-based screening test include a high prevalence of the disease being screened, a safe and cost-effective test, and a proven effective treatment for the detected disease (Table 1) [29]. NPs are attractive candidates to screen for CVD in the general population for several reasons [30]. First, the cardiovascular diseases that are detectable by elevated NP levels, including left ventricular dysfunction, cardiac dysrhythmias, and valvular disease, are common and cause significant morbidity and mortality. Second, NP levels are often elevated early on in the disease process of patients who go on to

Table 1 World Health Organization criteria for screening

The condition sought should be an important health problem for the individual and community

There should be an accepted treatment or useful intervention for patients with the disease

Facilities for diagnosis and treatment should be available

There should be a recognizable latent or early symptomatic stage

There should be a suitable and acceptable screening test

The natural history of the disease, including development from latent to declared disease, should be adequately understood

There should be an agreed policy on whom to treat as patients

The cost (including diagnosis and treatment of patients diagnosed) should be economically balanced in relation to possible expenditure on medical care as a whole

Case-finding should be a continuing process and not a "once and for all" project

(Adapted from Wilson [29].) develop left ventricular dysfunction as well as other cardiovascular conditions, allowing for timely detection of disease prior to symptom onset $[31 \bullet, 32]$. Third, early treatment of latent disease may improve outcomes; for example, early use of angiotensin-converting enzyme inhibitors may prevent the development of heart failure in patients with asymptomatic left ventricular dysfunction [33], and the development of arterial hypertension could be prevented with earlier initiation of angiotensin-receptor blocker therapy [34]. Finally, several studies have shown that, in the proper setting of targeted higher-risk populations, NPs may prove cost effective for screening for CVD [35-37].

Screening tests have better value in populations with a higher prevalence of disease. The value of NPs in assessing cardiovascular risk is bolstered by the fact that they can both detect and predict a variety of clinical disorders. When NPs are used to screen only for left ventricular dysfunction, results have been mixed [15, 38-43]. However, when used to screen for the presence of a variety of cardiovascular diseases [35, 44], or to assess risk across the broader spectrum of CVD, NPs have consistently emerged as strong predictors. An approach that capitalizes on both the screening and the risk assessment capabilities of NPs may have the greatest chance of improving care.

\section{Targeting Specific Subpopulations}

The ultimate success of NPs as a tool for risk assessment likely hinges upon identifying and targeting appropriate subpopulations. Risk assessment tools rarely are indicated for every individual regardless of age, gender, or other risk factors, even when the aim is primary prevention. Rather, by targeting groups with high-risk features, the accuracy and cost effectiveness of risk assessment can be improved. In the case of screening asymptomatic individuals with NPs, a strategy targeting moderate-risk or higher-risk groups has a much higher likelihood of success than does a shotgun approach of evaluating every single individual.

Individuals currently considered to be at moderate risk for CVD may stand to benefit the most from assessment with NPs. Examples include individuals with borderline or only single abnormalities of traditional risk factors (such as those with a family history of CVD but no other risk factors, those with isolated hypertension, or the elderly), those with a Framingham risk score of $10 \%$ to $20 \%$, and those with atypical symptoms of CVD. We have recently shown in a cohort of 829 outpatients referred for an echocardiogram that individuals who are at risk for heart failure but who are asymptomatic (American Heart Association/American College of Cardiology Stage A or B) with elevated BNP levels $(\geq 100 \mathrm{pg} / \mathrm{mL}$ ) are actually at equal or higher risk for future cardiovascular events than 
are individuals with a history of heart failure whose BNP is less than $100 \mathrm{pg} / \mathrm{mL}$ (Fig. 2) [45]. Thus, NPs may be useful to identify asymptomatic individuals at high risk for future cardiovascular events. In moderate-risk individuals who may be undecided about starting medications for primary prevention, a markedly abnormal NP level may tilt the balance in favor of treatment.

Individuals at highest risk for CVD in whom aggressive preventive treatment is already recommended, including those with chronic kidney disease, diabetes, or peripheral vascular disease, may actually be in less-attractive subgroups to measure NP levels for risk assessment, although a low NP level in these individuals could encourage a more simplified approach to future testing with a high negative predictive value [42]. Conversely, assessment of NP levels in very low-risk subgroups such as young, healthy individuals without any traditional risk factors is unlikely to prove economically attractive. However, if relatively inexpensive yet highly accurate screening panels become available that include NPs as one part of a multi-marker approach, even this paradigm could change.

\section{Recent Advances}

\section{Multi-Marker and Multi-Modality Approach}

Interest in a multi-marker (or multi-modality) approach to risk assessment is growing, as reflected by the number of recent studies evaluating such strategies. The rationale for combining multiple risk markers is compelling, as such an

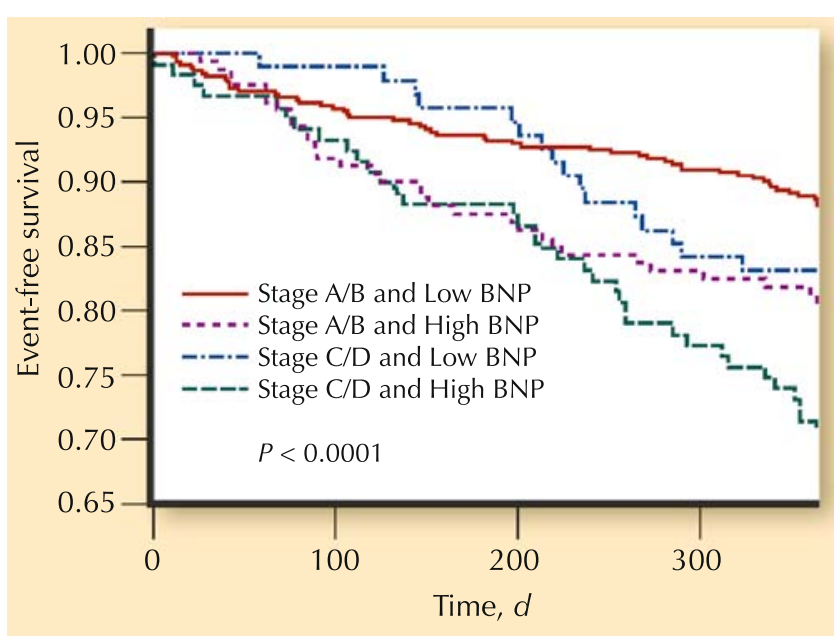

Fig. 2 Unadjusted event-free survival over 1 year in four groups based on American College of Cardiology/American Heart Association heart failure stage and B-type natriuretic peptide (BNP) level among 829 outpatients referred for an echocardiogram. High BNP is $\geq 100 \mathrm{pg} / \mathrm{mL}$ and low BNP is $<100 \mathrm{pg} / \mathrm{mL}$. The $P$ value is from the log-rank test for comparison across groups. (Adapted from Daniels et al. [45]; with permission.) approach can extract information from a variety of distinct pathophysiologic pathways, potentially broadening the scope and sensitivity of risk prediction [46]. We have only begun to scratch the surface on exploring combinations of biomarkers, genetic testing, and imaging or other noninvasive measures of risk. Along these lines, Shaw et al. [47•] recently showed that BNP and coronary artery calcium scores were each independent predictors of cardiovascular events in 2,458 asymptomatic adults recruited from a pool of general medicine outpatients (Fig. 3). In this study, BNP was the second strongest predictor of events (after coronary artery calcium score) over a median of 4 years of follow-up, even after accounting for the Framingham risk score. Combining distinct risk markers for risk prediction requires more study, but because of the complementary information provided, this approach is promising.

The importance of a multi-pronged approach is highlighted by several studies suggesting that NPs may be less robust markers in asymptomatic individuals for predicting CHD risk specifically, compared with its utility for predicting CVD risk. This was first suggested by the Framingham Offspring data, where NP levels were not significantly associated with the risk of CHD events, despite being associated with CVD in general (adjusted hazard ratios $[95 \% \mathrm{CI}$ per 1 standard deviation increase in BNP] of 1.10 [0.89-1.37; $P=0.37]$ for CHD events versus 1.28 [1.03-1.59; $P=0.03]$ for first major CVD event) [8]. In the past year, similar findings were reported by Tsuchida and Tanabe [28], who showed that despite an increased risk of cardiovascular events, cardiovascular mortality, all-cause mortality, heart failure, stroke, and atrial fibrillation among 3,123 consecutive outpatients with elevated BNP, there was no increased risk of CHD (hazard ratios for BNP level $\geq 100 \mathrm{pg} / \mathrm{mL}$ compared with BNP $<100 \mathrm{pg} / \mathrm{mL}$ were 4.6 [3.5-6.1; $P<0.0001]$ for CVD events versus $0.6[0.2-1.7$; $P=0.34]$ for $\mathrm{CHD}$ events). However, there is significant heterogeneity in studies that report the magnitude of the association between NP levels and CHD risk. Metaanalyses and several other recent studies have found that NP levels are in fact predictive of coronary events as well as CVD events in asymptomatic individuals, albeit often with slightly lower risk estimates [20, 21, 23, 26, 48, 49]. Melander et al. [22] demonstrated that of six novel and conventional biomarkers studied in 5,067 community-based Swedish participants without CVD, only NT-proBNP was predictive of both CVD and CHD events.

In studies evaluating multi-marker approaches to CVD risk assessment, NPs consistently emerge as important contributors [22, 24, 50]. However, in several of these studies the combination of multiple biomarkers only marginally increased the $\mathrm{C}$-statistic for risk prediction [22, 50]. Some researchers have therefore concluded that the use of multiple biomarkers, as studied to date, has added only 


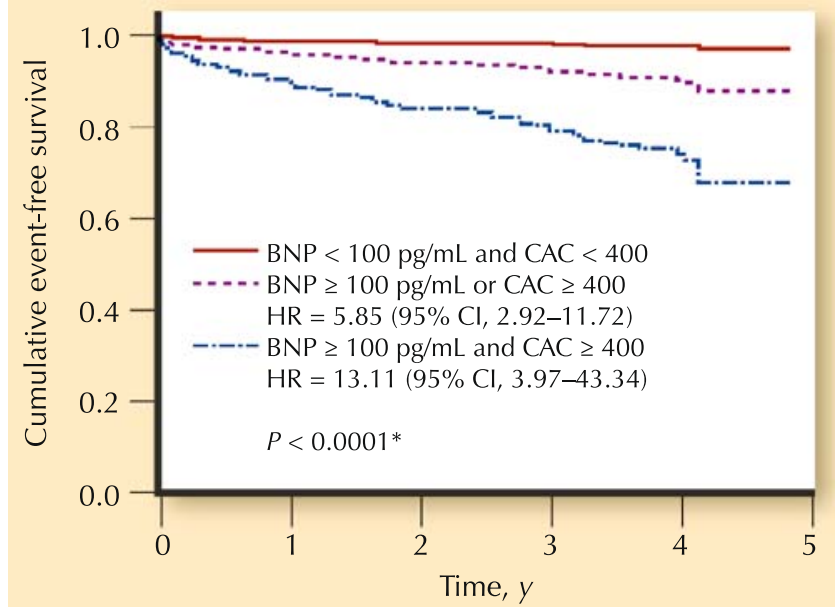

Fig. 3 Cumulative cardiovascular event-free survival in patients with combined B-type natriuretic peptide (BNP) and coronary artery calcium (CAC) measurements. HR hazard ratio. (Adapted from Shaw et al. [47•]; with permission.)

moderately to overall risk prediction based on traditional cardiovascular risk factors [51]. On the other hand, relying solely on the C-statistic may have limited utility for assessing future risk in a currently healthy population [52]. The final words are far from written on the optimal combination of risk markers and on the specifics regarding the best way to utilize a multi-marker approach to assess asymptomatic individuals.

\section{Preoperative Risk Assessment}

Several recent publications have evaluated NPs for assessing preoperative risk. In a meta-analysis of nine studies including a total of 3,281 patients undergoing noncardiac surgery, Karthikeyan et al. [53] examined the association between elevated preoperative levels of NPs with major adverse cardiac events within 30 days. All nine studies showed an association between elevated preoperative NP levels and adverse cardiac outcomes, with a pooled odds ratio of $19.3(95 \% \mathrm{CI}, 8.5-43.7)$ after adjusting for other risk factors. Ryding et al. [54] had strikingly similar findings in their meta-analysis, which included 15 publications comprising 4,856 patients undergoing noncardiac surgery (odds ratio of 19.8; 95\% CI, 13.2-29.7), whereas Rodseth et al. [55] showed the same in a meta-analysis of 1,127 vascular surgery patients (odds ratio of $17.4 ; 95 \% \mathrm{CI}$, 3.3-91.2). These and other studies have also shown the converse (ie, patients with normal preoperative NP levels had a very low risk of adverse cardiac events) [55-57]. This has raised speculation that an NP-guided algorithm for preoperative risk assessment may be cost effective [58], whereby individuals with normal NP levels could potentially proceed directly to surgery without further expensive invasive and noninvasive testing, whereas those with elevated levels may benefit from further risk stratification and optimization of medications plus more intensive intraoperative and perioperative monitoring.

\section{Screening for Left Ventricular Dysfunction}

The purpose of screening tests is to identify cardiac functional and structural abnormalities at an early stage. Over the past decade, NP levels have been evaluated as a tool to screen asymptomatic individuals for left ventricular dysfunction in a variety of settings [40, 59, 60]. Recent studies shed further light on this area. In 2009, Betti et al. [42] showed in 1,012 asymptomatic individuals at high risk for heart failure because of diabetes and/or hypertension that the cut-point of NT-proBNP less than $125 \mathrm{pg} / \mathrm{mL}$ had an excellent negative predictive value of nearly $100 \%$ for ruling out asymptomatic left ventricular dysfunction. If this finding results in fewer echocardiograms for high-risk patients such as these, NP testing could prove cost effective in this situation.

Despite the high negative-predictive value among highrisk individuals, the utility of NPs as a screening tool for left ventricular dysfunction in the community has often proved suboptimal. de Lemos et al. [15•] systematically evaluated false-positive and false-negative screening evaluations in 2,429 population-based participants from the Dallas Heart Study. Characteristics associated with falsenegative NP values included younger age, lower left ventricular mass, higher left ventricular ejection fraction, and a trend toward better renal function; characteristics associated with false-positive NP values included older age, poorer renal function, higher prevalence of coronary artery calcification, and greater left ventricular mass.

Although NPs did not accurately discriminate individuals with left ventricular systolic dysfunction overall, performance among high-risk men (age $\geq 50$ years or with hypertension) was comparable to other commonly used screening tests such as prostate-specific antigen screening for prostate cancer, Papanicolaou smears for cervical cancer, and mammography for breast cancer (area under the curve of 0.70 and 0.85 , respectively) [15•, 61-63]. Furthermore, as described by the authors, previous population-based studies that have also shown suboptimal performance of NPs for screening for left ventricular dysfunction have subsequently shown that high levels are nonetheless predictive of worse cardiovascular outcomes. This suggests that NP levels reflect more than the structural and functional abnormalities that are seen on echocardiogram. Further support comes from a recent study by Mogelvang et al. [31•] in which 1,012 community-based participants underwent echocardiography. They found that preclinical systolic and diastolic dysfunction, as assessed by tissue Doppler imaging, was associated with elevated 
plasma proBNP levels, even when conventional echocardiography was normal. NPs seem to represent a synthesized measure of an individual's cardiovascular risk, which, in addition to reflecting overt cardiac abnormalities, also reflects physiologic perturbations of minor structural and functional cardiac abnormalities as well as other factors, such as renal dysfunction [15•].

\section{Controversies and Future Directions}

One difficulty inherent to any approach that uses biomarkers to stratify risk is how to choose the optimal cut-point for action. Choosing a cut-point makes a binary variable out of a continuous one, and although this greatly simplifies clinical use, a significant amount of information is lost in the translation. Furthermore, the tension between sensitivity and specificity is a transcendent one in cutpoint selection (as attested to by the frequently changing guidelines for low-density lipoprotein cholesterol targets, systolic blood pressure goals, and the fasting glucose level needed for a diagnosis of diabetes.) Choosing an ideal NP cut-point is even more complicated due to intra-individual variability as well as the variability in baseline levels based on the population being screened. This makes it unlikely that any single cut-point will be sufficiently acceptable across all populations.

Future studies evaluating NPs (or any new marker or multi-marker panel) for risk assessment will benefit from incorporating several newer statistical methods that have been developed and recommended for gauging the information provided by a new risk marker $[64,65 \cdot 0]$. In addition to conventional analyses such as proportional hazards models, newer performance criteria include measures of discrimination, calibration, validation, and reclassification. However, statistical methods alone cannot measure the full clinical value of the information provided by a new risk marker. The goal of improving risk assessment is to improve how we respond to various risk categories; therefore, the effect of the risk marker on clinical decisions and clinical outcomes is ultimately the data in which we should be most interested.

\section{Conclusions}

There are insufficient data at the present time to recommend a strategy of NP-guided risk assessment for asymptomatic individuals in the population at large. However, accumulating evidence suggests that an NP-guided approach may make a positive impact in select subpopulations, including those at intermediate or higher risk for CVD and those undergoing preoperative evaluation. While we await costeffectiveness and outcome studies to confirm these uses of
NPs, investigations will continue to search for the best recipe of other subgroups that may benefit, plus new markers and combinations of risk markers that can make assessing cardiovascular risk ever more accurate.

Acknowledgment This work is supported in part by a grant from the American Heart Association National Affiliate.

Disclosure Dr. Daniels has received research funding from Roche Diagnostics and Biosite, Inc.

Open Access This article is distributed under the terms of the Creative Commons Attribution Noncommercial License which permits any noncommercial use, distribution, and reproduction in any medium, provided the original author(s) and source are credited.

\section{References}

Papers of particular interest, published recently, have been highlighted as:

- Of importance

•. Of major importance

1. Pearson TA, Blair SN, Daniels SR, et al.: AHA Guidelines for Primary Prevention of Cardiovascular Disease and Stroke: 2002 Update: Consensus Panel Guide to Comprehensive Risk Reduction for Adult Patients Without Coronary or Other Atherosclerotic Vascular Diseases. American Heart Association Science Advisory and Coordinating Committee. Circulation 2002, 106:388-391.

2. Mosca L, Banka CL, Benjamin EJ, et al.: Evidence-based guidelines for cardiovascular disease prevention in women: 2007 update. J Am Coll Cardiol 2007, 49:1230-1250.

3. Vasan RS, Sullivan LM, Wilson PW, et al.: Relative importance of borderline and elevated levels of coronary heart disease risk factors. Ann Intern Med 2005, 142:393-402. (Published erratum appears in Ann Intern Med 2005, 142:681.)

4. Greenland P, Knoll MD, Stamler J, et al.: Major risk factors as antecedents of fatal and nonfatal coronary heart disease events. JAMA 2003, 290:891-897.

5. Magnus P, Beaglehole R: The real contribution of the major risk factors to the coronary epidemics: time to end the "only-50\%" myth. Arch Intern Med 2001, 161:2657-2660.

6. Khot UN, Khot MB, Bajzer CT, et al.: Prevalence of conventional risk factors in patients with coronary heart disease. JAMA 2003, 290:898-904.

7. Daniels LB, Maisel AS: Natriuretic peptides. J Am Coll Cardiol 2007, 50:2357-2368.

8. Wang TJ, Larson MG, Levy D, et al.: Plasma natriuretic peptide levels and the risk of cardiovascular events and death. N Engl J Med 2004, 350:655-663.

9. Daniels LB, Laughlin GA, Clopton P, et al.: Minimally elevated cardiac troponin $\mathrm{T}$ and elevated $\mathrm{N}$-terminal pro-B-type natriuretic peptide predict mortality in older adults: results from the Rancho Bernardo Study. J Am Coll Cardiol 2008, 52:450-459.

10. National Cholesterol Education Prgram (NCEP) Expert Panel on Detection, Evaluation, and Treatment of High Blood Cholesterol in Adults (Adult Treatment Panel III): Third Report of the National Cholesterol Education Program (NCEP) Expert Panel on Detection, Evaluation, and Treatment of High Blood Choles- 
terol in Adults (Adult Treatment Panel III) final report. Circulation 2002, 106:3143-1421.

11. Wilson PW, D'Agostino RB, Levy D, et al.: Prediction of coronary heart disease using risk factor categories. Circulation 1998, 97:1837-1847.

12. Rose G: Sick individuals and sick populations. Int J Epidemiol 1985, 14:32-38.

13. Jackson R, Lawes CM, Bennett DA, et al.: Treatment with drugs to lower blood pressure and blood cholesterol based on an individual's absolute cardiovascular risk. Lancet 2005, 365:434 441.

14. Sibley C, Blumenthal RS, Merz CN, Mosca L: Limitations of current cardiovascular disease risk assessment strategies in women. J Womens Health (Larchmt) 2006, 15:54-56.

15. - de Lemos JA, McGuire DK, Khera A, et al.: Screening the population for left ventricular hypertrophy and left ventricular systolic dysfunction using natriuretic peptides: results from the Dallas Heart Study. Am Heart J 2009, 157:746-753.e2. This population-based study of 2429 individuals assessed the utility of NPs to screen for left ventricular dysfunction or left ventricular hypertrophy, and systematically determined the factors associated with false-positive and false-negative values.

16. Hobbs FD, Davis RC, Roalfe AK, et al.: Reliability of N-terminal proBNP assay in diagnosis of left ventricular systolic dysfunction within representative and high risk populations. Heart 2004, 90:866-870.

17. McKie PM, Rodeheffer RJ, Cataliotti A, et al.: Amino-terminal pro-B-type natriuretic peptide and B-type natriuretic peptide: biomarkers for mortality in a large community-based cohort free of heart failure. Hypertension 2006, 47:874-880.

18. Hammerer-Lercher A, Neubauer E, Muller S, et al.: Head-to-head comparison of $\mathrm{N}$-terminal pro-brain natriuretic peptide, brain natriuretic peptide and $\mathrm{N}$-terminal pro-atrial natriuretic peptide in diagnosing left ventricular dysfunction. Clin Chim Acta 2001, 310:193-197.

19. Mueller T, Gegenhuber A, Poelz W, Haltmayer M: Head-to-head comparison of the diagnostic utility of BNP and NT-proBNP in symptomatic and asymptomatic structural heart disease. Clin Chim Acta 2004, 341:41-48.

20. Kistorp C, Raymond I, Pedersen F, et al.: N-terminal pro-brain natriuretic peptide, C-reactive protein, and urinary albumin levels as predictors of mortality and cardiovascular events in older adults. JAMA 2005, 293:1609-1616.

21. Laukkanen JA, Kurl S, Ala-Kopsala M, et al.: Plasma N-terminal fragments of natriuretic propeptides predict the risk of cardiovascular events and mortality in middle-aged men. Eur Heart J 2006, 27:1230-1237.

22. Melander O, Newton-Cheh C, Almgren P, et al.: Novel and conventional biomarkers for prediction of incident cardiovascular events in the community. JAMA 2009, 302:49-57.

23. Olsen MH, Hansen TW, Christensen MK, et al.: N-terminal probrain natriuretic peptide, but not high sensitivity C-reactive protein, improves cardiovascular risk prediction in the general population. Eur Heart J 2007, 28:1374-1381.

24. Zethelius B, Berglund L, Sundstrom J, et al.: Use of multiple biomarkers to improve the prediction of death from cardiovascular causes. N Engl J Med 2008, 358:2107-2116.

25. Groenning BA, Raymond I, Hildebrandt PR, et al.: Diagnostic and prognostic evaluation of left ventricular systolic heart failure by plasma $\mathrm{N}$-terminal pro-brain natriuretic peptide concentrations in a large sample of the general population. Heart 2004, 90:297303.

26. De Sutter J, De Bacquer D, Cuypers S, et al.: Plasma N-terminal pro-brain natriuretic peptide concentration predicts coronary events in men at work: a report from the BELSTRESS study. Eur Heart J 2005, 26:2644-2649.
27. Ueda R, Yokouchi M, Suzuki T, et al.: Prognostic value of high plasma brain natriuretic peptide concentrations in very elderly persons. Am J Med 2003, 114:266-270.

28. Tsuchida K, Tanabe K: Plasma brain natriuretic peptide concentrations and the risk of cardiovascular events and death in general practice. J Cardiol 2008, 52:212-223.

29. Wilson JM: The evaluation of the worth of early disease detection. J R Coll Gen Pract 1968, 16(Suppl 2):48-57.

30. Daniels LB, Maisel A: B-type natriuretic peptide: time to incorporate natriuretic peptides in our practice. J Cardiovasc Med (Hagerstown) 2006, 7:414-415.

31. • Mogelvang R, Goetze JP, Pedersen SA, et al.: Preclinical systolic and diastolic dysfunction assessed by tissue doppler imaging is associated with elevated plasma pro-B-type natriuretic peptide concentrations. J Card Fail 2009, 15:489-495. This communitybased study shows that natriuretic peptide levels can be elevated in individuals with very early ventricular dysfunction that is not manifest on conventional echocardiography, perhaps explaining the reason for elevated BNP levels in some asymptomatic individuals with apparently normal hearts.

32. Gerber IL, Legget ME, West TM, et al.: Usefulness of serial measurement of $\mathrm{N}$-terminal pro-brain natriuretic peptide plasma levels in asymptomatic patients with aortic stenosis to predict symptomatic deterioration. Am J Cardiol 2005, 95:898-901.

33. Effect of enalapril on mortality and the development of heart failure in asymptomatic patients with reduced left ventricular ejection fractions. The SOLVD Investigators. N Engl J Med 1992, 327:685-691. (Published erratum appears in N Engl J Med 1992, 327:1768.)

34. Julius S, Nesbitt SD, Egan BM, et al.: Feasibility of treating prehypertension with an angiotensin-receptor blocker. N Engl J Med 2006, 354:1685-1697.

35. Nakamura M, Endo H, Nasu M, et al.: Value of plasma B type natriuretic peptide measurement for heart disease screening in a Japanese population. Heart 2002, 87:131-135.

36. Nielsen OW, McDonagh TA, Robb SD, Dargie HJ: Retrospective analysis of the cost-effectiveness of using plasma brain natriuretic peptide in screening for left ventricular systolic dysfunction in the general population. J Am Coll Cardiol 2003, 41:113-120.

37. Heidenreich PA, Gubens MA, Fonarow GC, et al.: Costeffectiveness of screening with B-type natriuretic peptide to identify patients with reduced left ventricular ejection fraction. $\mathrm{J}$ Am Coll Cardiol 2004, 43:1019-1026.

38. Redfield MM, Rodeheffer RJ, Jacobsen SJ, et al.: Plasma brain natriuretic peptide to detect preclinical ventricular systolic or diastolic dysfunction: a community-based study. Circulation 2004, 109:3176-3181.

39. Vasan RS, Benjamin EJ, Larson MG, et al.: Plasma natriuretic peptides for community screening for left ventricular hypertrophy and systolic dysfunction: the Framingham heart study. JAMA 2002, 288:1252-1259.

40. McDonagh TA, Robb SD, Murdoch DR, et al.: Biochemical detection of left-ventricular systolic dysfunction. Lancet 1998, 351:9-13

41. Luchner A, Burnett JC Jr, Jougasaki M, et al.: Evaluation of brain natriuretic peptide as marker of left ventricular dysfunction and hypertrophy in the population. J Hypertens 2000, 18:11211128.

42. Betti I, Castelli G, Barchielli A, et al.: The role of N-terminal PRO-brain natriuretic peptide and echocardiography for screening asymptomatic left ventricular dysfunction in a population at high risk for heart failure. The PROBE-HF study. J Card Fail 2009, 15:377-384.

43. Costello-Boerrigter LC, Boerrigter G, Redfield MM, et al.: Amino-terminal pro-B-type natriuretic peptide and B-type natri- 
uretic peptide in the general community: determinants and detection of left ventricular dysfunction. J Am Coll Cardiol 2006, 47:345-353.

44. Struthers AD: Introducing a new role for BNP: as a general indicator of cardiac structural disease rather than a specific indicator of systolic dysfunction only. Heart 2002, 87:97-98.

45. Daniels LB, Clopton P, Jiang K, et al.: Prognosis of stage A or B heart failure patients with elevated B-type natriuretic peptide levels. J Card Fail 20010 (in press).

46. Daniels LB: Multiple biomarker assessment in primary prevention of cardiovascular disease. Curr Cardiov Risk Rep 2009, 3:131136.

47. - Shaw LJ, Polk DM, Kahute TA, et al.: Prognostic accuracy of Bnatriuretic peptide measurements and coronary artery calcium in asymptomatic subjects (from the Early Identification of Subclinical Atherosclerosis by Noninvasive Imaging Research [EISNER] study). Am J Cardiol 2009, 104:1245-1250. This study evaluated 2458 asymptomatic adult outpatients and showed that BNP and coronary artery calcium scores were independent predictors of cardiovascular events. It lends support to the notion that a multimodality approach to risk assessment could improve upon current methods.

48. Olsen MH, Wachtell K, Nielsen OW, et al.: N-terminal brain natriuretic peptide predicted cardiovascular events stronger than high-sensitivity C-reactive protein in hypertension: a LIFE substudy. J Hypertens 2006, 24:1531-1539.

49. Astor BC, Yi S, Hiremath L, et al.: N-terminal prohormone brain natriuretic peptide as a predictor of cardiovascular disease and mortality in blacks with hypertensive kidney disease: the African American Study of Kidney Disease and Hypertension (AASK). Circulation 2008, 117:1685-1692.

50. Wang TJ, Gona P, Larson MG, et al.: Multiple biomarkers for the prediction of first major cardiovascular events and death. N Engl J Med 2006, 355:2631-2639.

51. Ware JH: The limitations of risk factors as prognostic tools. N Engl J Med 2006, 355:2615-2617.

52. Cook NR: Use and misuse of the receiver operating characteristic curve in risk prediction. Circulation 2007, 115:928-935.

53. Karthikeyan G, Moncur RA, Levine O, et al.: Is a pre-operative brain natriuretic peptide or $\mathrm{N}$-terminal pro-B-type natriuretic peptide measurement an independent predictor of adverse cardiovascular outcomes within 30 days of noncardiac surgery? A systematic review and meta-analysis of observational studies. J Am Coll Cardiol 2009, 54:1599-1606.
54. Ryding AD, Kumar S, Worthington AM, Burgess D: Prognostic value of brain natriuretic peptide in noncardiac surgery: a metaanalysis. Anesthesiology 2009, 111:311-319.

55. Rodseth RN, Padayachee L, Biccard BM: A meta-analysis of the utility of pre-operative brain natriuretic peptide in predicting early and intermediate-term mortality and major adverse cardiac events in vascular surgical patients. Anaesthesia 2008, 63:1226-1233.

56. Bolliger D, Seeberger MD, Lurati Buse GA, et al.: A preliminary report on the prognostic significance of preoperative brain natriuretic peptide and postoperative cardiac troponin in patients undergoing major vascular surgery. Anesth Analg 2009, 108:1069-1075.

57. Dernellis J, Panaretou M: Assessment of cardiac risk before noncardiac surgery: brain natriuretic peptide in 1590 patients. Heart 2006, 92:1645-1650.

58. Bolliger D, Seeberger MD, Filipovic M: Pre-operative cardiac risk assessment in noncardiac surgery: are natriuretic peptides the magic bullet? J Am Coll Cardiol 2009, 54:1607-1608.

59. Galasko GI, Lahiri A, Barnes SC, et al.: What is the normal range for N-terminal pro-brain natriuretic peptide? How well does this normal range screen for cardiovascular disease? Eur Heart J 2005, 26:2269-2276.

60. Wang TJ, Levy D, Benjamin EJ, Vasan RS: The epidemiology of "asymptomatic" left ventricular systolic dysfunction: implications for screening. Ann Intern Med 2003, 138:907-916.

61. Schroder F, Kattan MW: The comparability of models for predicting the risk of a positive prostate biopsy with prostate-specific antigen alone: a systematic review. Eur Urol 2008, 54:274-290.

62. Swets JA, Getty DJ, Pickett RM, et al.: Enhancing and evaluating diagnostic accuracy. Med Decis Making 1991, 11:9-18.

63. Fahey MT, Irwig L, Macaskill P: Meta-analysis of Pap test accuracy. Am J Epidemiol 1995;141:680-689.

64. Pencina MJ, D'Agostino RB Sr, D'Agostino RB Jr, Vasan RS: Evaluating the added predictive ability of a new marker: from area under the ROC curve to reclassification and beyond. Stat Med 2008, 27:157-172; discussion 207-212.

65. • Hlatky MA, Greenland P, Arnett DK, et al.: Criteria for evaluation of novel markers of cardiovascular risk: a scientific statement from the American Heart Association. Circulation 2009, 119:2408-2416. (Published erratum appears in Circulation 2009, 119:e606.) This scientific statement reviews current concepts of risk evaluation and proposes standards for the critical appraisal of risk assessment methods with a focus on novel markers of CVD risk. 